\title{
A PRODUCT FORMULA FOR AN ARF-KERVAIRE INVARIANT
}

\author{
BY EDGAR H. BROWN, JR. ${ }^{1}$
}

Communicated April 15, 1974

In [1] we introduced an Arf-Kervaire type of invariant $\sigma(M) \in Z_{8}=Z / 8 Z$ defined for closed compact, even-dimensional manifolds $M$ having a certain kind of orientation (see below). In this announcement we give a product formula for $\sigma$. Our results are applicable to Poincaré duality spaces, but for simplicity we give them for smooth manifolds. A special case of our formula was given in [2].

Let $v^{m}$ be the map

$$
v^{m}=\prod v_{i}: B O_{k} \rightarrow \prod_{2 i>m} K\left(Z_{2}, i\right),
$$

where $v_{i} \in H^{i}\left(B O_{k}\right)$ is the $i$ th $\mathrm{Wu}$ class. Let $B O_{k}^{m}$ be the fibration over $B O_{k}$ induced by $v^{m}$ from the contractible fibration. Let $\zeta_{k}$ be the universal $k$-plane bundle over $B O_{k}$, and let $\zeta_{k}^{m}=p^{*} \zeta_{k}$, where $p: B O_{k}^{m} \rightarrow B O_{k}$ is the projection. The Whitney sum map, $\zeta_{k} \times \zeta_{l} \rightarrow \zeta_{k+l}$, lifts to a map $\mu: \zeta_{k}^{m} \times$ $\zeta_{l}^{n} \rightarrow \zeta_{k+l}^{m+n}$.

If $M$ is an $m$-manifold, a $W u$ orientation of $M$ is a bundle map $V: \nu \rightarrow \zeta_{k}^{m}$, where $\nu$ is the normal bundle of $M \subset R^{m+k}$. (Every manifold has a Wu orientation.) If $U$ and $V$ are Wu orientations on $M$ and $N, M \times N$ has a product orientation $U \times V$ defined in the obvious way. (For a detailed account of these ideas see [2].) Hereafter, manifold means a compact, closed, smooth manifold with a Wu orientation. $M \times N$ denotes the product manifold with the product orientation. The definition of $\sigma$ given in [1] is applicable to $M$, with its $\mathrm{Wu}$ orientation, if $\operatorname{dim} M=2 n$. Let $\sigma(M)=0$ if $\operatorname{dim} M=2 n+1$. The definition of $\sigma$ in [1] depended on a choice $\lambda_{n}: \pi_{2 n+k}\left(T\left(\zeta_{k}^{2 n}\right) \wedge K\left(Z_{2}, n\right)\right) \rightarrow Z_{4}$. Choose such $\lambda_{n}$ 's for each $n$ (such that $\lambda_{n}\left(\alpha_{n}\right)=2$ in the notation of [1]). $\left(\lambda_{2 n}\right.$ can and should be chosen so that $\sigma(M)=\operatorname{index}(M) \bmod 8$ if $M$ is an oriented (in the usual sense) $4 n$ manifold.) Since we killed $v_{n+1}$ to form $B O_{k}^{n}, S^{n}$ has a nontrivial $\mathrm{Wu}$ orientation. Let $\bar{S}^{n}$ denote $S^{n}$ with this orientation. It turns out that

AMS (MOS) subject classifications (1970). Primary 57A25, 57D65.

1 The author was supported by NSF grant GP-38920X and a Science Research Council of Britain Fellowship while carrying out this research. 
$\sigma\left(\bar{S}^{n} \times M\right) \in\{0,4\}$. Let $\sigma^{n}(M) \in Z_{2}, \quad n>0$, be defined by $4 \sigma^{n}(M)=$ $\sigma\left(\bar{S}^{n} \times M\right)$, where $4: Z_{2} \rightarrow Z_{8}$ is the inclusion.

THEOREM 1.1. The maps $\mu: \zeta_{k}^{m} \times \zeta_{l}^{n} \rightarrow \zeta_{k+l}^{m+n}$ can be chosen so that the following formulas hold:

$$
\begin{aligned}
\sigma(M \times N) & =\sigma(M) \sigma(N)+\sum_{n} 4\left(\sigma^{n}(M) \sigma^{n}(N)\right), \\
\sigma^{n}(M \times N) & =\sigma(M) \sigma^{n}(N)+\sigma^{n}(M) \sigma(N) .
\end{aligned}
$$

REMARK. $\sigma^{n}(M)=0$ for $n>\operatorname{dim} M$ or $n+\operatorname{dim} M$ odd. In the above formula, $\sigma(M) \sigma^{n}(N)$ means $(\sigma(M) \bmod 2) \sigma^{n}(N)$. In [1] it was shown that $\sigma(M)=$ Euler characteristic of $M$ modulo 2 .

THEOREM 1.2. If $m=\operatorname{dim} M, m-n$ is even and $v_{(m-n) / 2}(M)=0$, then $\sigma^{n}(M)=0 ; \sigma^{n}\left(\bar{S}^{n}\right)=1\left(v_{0}=1\right) ; \sigma\left(\bar{S}^{n} \times \bar{S}^{n}\right)=4 ; \sigma^{n}\left(\bar{S}^{n} \times R P^{2 m}\right)=1$.

A somewhat more amusing way of writing Theorem 1.1 is as follows: Let $A=Z_{8}\left[a_{n}\right], n=1,2, \cdots$, modulo the relations $2 a_{n}=a_{n} a_{m}=0, n \neq m$, $a_{n}^{2}=4$. Let $\sum(M)=\sigma(M)+\sum \sigma^{n}(M) a_{n}$.

THEOREM 1.3. $\sum(M \times N)=\sum(M) \sum(N)$.

\section{BIBLIOGRAPHY}

1. E. H. Brown, Jr., Generalizations of the Kervaire invariant, Ann. of Math. (2) 95 (1972), 368-383. MR 45 \#2719.

2. W. Browder, The Kervaire invariant, products and Poincaré transversality, Topology 12 (1973), 145-157.

Department of Mathematics, Brandeis University, Waltham, Massachusetts 02154 